\title{
FACTORES SOCIO-AFECTIVOS RELACIONADOS CON LAS DIFICULTADES ESCOLARES EN NIÑAS Y NIÑOS "ESTRELLA" DEL PROGRAMA PSICOMOTRICIDAD E INTERVENCIÓN
}

\author{
Emmanuel Herrera González, Laura Delgado Tenorio, Héctor Fonseca \\ Schmidt, Pilar Vargas Ramírez. \\ Programa de Psicomotricidad (PSICOMI), Escuela de Ciencias del \\ Movimiento Humano y la calidad de Vida, Facultad de Ciencias de la \\ Salud, Universidad Nacional, Heredia, Costa Rica. \\ emmahe21@yahoo.com
}

\section{RESUMEN}

\begin{abstract}
El presente estudio muestra los principales factores socio-afectivos relacionados con las dificultades escolares de un grupo de tres niñas y tres niños identificados como "estrella" por el Programa Psicomotricidad e Intervención. El mismo se desarrolló a través de una metodología mixta, en la que se aplicaron el Test de Figura Humana, el Dibujo Kinético de la Familia y una entrevista a la madre de cada estudiante. Los resultados revelaron la existencia de factores conflictivos familiares que afectan el estado emocional de las y los niños, generándoles sentimientos negativos consigo mismos que terminan afectado sus interacciones sociales y también su desempeño escolar.
\end{abstract}

PALABRAS CLAVES: Factores socio-afectivos, niñas y niños “estrella”.

\begin{abstract}
This study shows the main socio- affective factors related to school difficulties of a group of three girls and three boys identified as "star" in the Programa Psicomotricidad e Intervención (Psychomotor and Intervention Program). The study was developed through a mixed methodology, in which the Human Figure Test, the Kinetic Family Drawing and an interview with the mother of each student were applied. The most important results revealed the existence of family conflicting factors that affect the emotional state of children, generating negative feelings about themselves that affect their social interactions and their school performance.
\end{abstract}

KEY WORDS: Socio-affective factors, "star” children.

Revista MHSalud® (ISSN: 1659-097X) Vol. 9. No. 1. Enero-Julio, 2012

https://doi.org/10.15359/mhs.9-1.2 
URL www.una.ac.cr/mhsalud

\section{INTRODUCCIÓN:}

Dentro de la antigua Escuela Ciencias del Deporte de la Universidad Nacional, se realizó el Programa Identificación Temprana, el cual desarrolló, durante los años 1986 y 1996, un diagnóstico perceptual-motor denominado Sistema Peques. Los resultados alcanzados hasta ahora, demostraron que la aplicación total del Sistema PEQUES predice, con un alto grado de certeza, cuáles serán los niños y niñas que tienen mayores probabilidades de aprobar o repetir un año escolar en I Ciclo. Lo anterior confirma que el diseño de procedimientos en la aplicación de los instrumentos del Sistema Peques, así como su calificación y la posterior interpretación de los resultados fueron estadísticamente $(\mathrm{p}<0.01)$ eficaces. Al realizar la valoración en el primer semestre de preescolar, permitió clasificar correctamente el éxito o el fracaso escolar de un $86 \%$ de los niños(as) que cursaron la escuela hasta el tercer año, específicamente el $87 \%$ de los repitientes y el $86 \%$ de los aprobados, superando otros estudios.

Como continuidad del mismo, se crea el Programa Psicomotricidad e Intervención (PSICOMI) de la Escuela de Ciencias del Movimiento Humano y Calidad de Vida de la Universidad Nacional, en el año 2008, dentro del cual se realiza el diagnóstico perceptual motor y se interviene desde el paradigma de la psicomotricidad, procurando fomentar el desarrollo integral del niño y la niña, contemplando el área motora, cognitiva y socioafectiva del mismo/a, todo ello mediante la labor interdisciplinaria de profesionales en educación física, psicopedagogía, educación y psicología.

En el marco de dicho Programa, se realizó entre los años 2008 y 2010, un diagnóstico en el cual se identificaron tres niñas y tres niños “estrella” de la Escuela Villalobos, en Lagunilla de Heredia, que presentaban dificultades a nivel escolar. A partir de las evaluaciones realizadas fue posible identificar una serie de factores socio-afectivos que se encontraban vinculados a dichas dificultades escolares, presentándose como un problema u obstáculo para el logro de sus metas académicas.

De esta forma, el presente artículo pretende evidenciar aquellos factores socio-afectivos que se encontraron vinculados a las dificultades escolares de las y los niños que participaron en el Programa Psicomotricidad e Intervención, por su condición de riesgo a perder el año escolar, ello con el objetivo de analizar los factores socio-afectivos relacionados con las dificultades escolares en las niñas y niños “estrella” del Programa Psicomotricidad e Intervención (PSICOMI).

\section{Marco teórico:}

Los centros educativos formales están dirigidos a "niños normales o promedio" (Jadue, 2002), pretendiendo realizar una homogenización del estudiantado, omitiendo las diferencias y generando que la niña o el niño que por alguna razón se salga de la "norma"

Revista MHSalud® (ISSN: 1659-097X) Vol. 9. No. 1. Enero-Julio, 2012 
presente riesgo de bajo rendimiento y predisposición a experiencias negativas en el medio escolar.

Siendo así, las y los estudiantes que presentan diferencias notables en relación con el promedio de su edad, son rezagados, generando múltiples deserciones pues el sistema formal no está acondicionado para dar respuesta a dicha diversidad. De manera inusual, él o la estudiante puede obtener fuera del centro educativo una valoración clínica de su condición de diferencia, y podría aplicársele una adecuación curricular ó enviarlo a un centro de educación especial, más en el sistema donde se encuentra no se harán mayores ajustes, pues está pensado y estructurado para la homogeneidad.

Así, para el año 2000, el Estado de la Nación (citado por Woodburn, Fernández y Bochini, 2007) reportó 45.000 repitentes en las escuelas de Costa Rica, de quienes 15.000 habían perdido el primer año escolar. Para el año 2006, se reportaban 12.650 niñas y niños de primer grado que habían reprobado, significando ello el $12.8 \%$ de la matricula total de dicho nivel, evidenciando la magnitud de esta problemática escolar. Para el año 2009 se encuentran 9361 niñas y niños repitentes en primer nivel (Estado de la Nación, 2009).

De igual manera, para el año 2010, 29.550 niñas y niñas habían reprobado el año que cursaban, representando esto un $6 \%$ de la matrícula inicial, de acuerdo con ello, el Departamento de Análisis Estadístico del Ministerio de Educación Pública aseguró que entre el periodo 1990 - 2010, la mayor cantidad de repitentes en I y II ciclo, eran de primer grado, cantidad equivalente al 13.5\%, lo que significó un aumento en las cifras.

Es así que se reflejan las dificultades que la acción estatal ha tenido para solucionar dicha problemática, dentro de las cuales, no se han desarrollado estrategias específicas que contemplen el análisis de los factores socio-afectivos más importantes en la vida de las y los estudiantes que se están vinculados a su formación educativa.

Sin embargo, se demuestra en investigaciones como las realizadas por Lozano y García (2000), Trianes y García (2002), Luchini (2002), y muy especialmente la elaborada por Espinoza, Herrera y Portuguez (2009), en el contexto escolar costarricense, la existencia de factores socio-afectivos estrechamente vinculados con el proceso de aprendizaje de las niñas y los niños, que pueden verse involucrados en la génesis de muchos problemas que obstaculizan el desarrollo escolar.

De acuerdo con ello, la Asesoría Nacional en coordinación con la Coalición Nacional de Salud Mental y Educación Especial de Estados Unidos (citada por Murillo, 2005), han considerado que existen problemas que "afectan el desempeño del estudiante en el contexto enseñanza- aprendizaje y su vida cotidiana, en forma perseverante e intensa, atribuyéndose su origen a alternaciones psíquicas por factores multicausales como relaciones familiares, aspectos socioculturales, factores orgánicos, relaciones sociales, abordajes y factores educativos, entre otros” (pp. 18-19), lo que refuerza la anterior afirmación. 
Tal consideración muestra la relación dialéctica entre las dificultades escolares y los factores socio-afectivos, en la cual se afectan mutuamente en el contexto educacional, como lo reflejan Trianes y García (2002), pues el niño y la niña, como seres integrales, se encuentran en interacción con sus pares, sus docentes y demás personal de la institución, así como con la comunidad en que viven y por supuesto con su familia, impactando todo ello en su estado emocional.

No obstante, para considerar tal relación como una problemática, la influencia negativa de los factores socio-afectivos en el rendimiento escolar debe ser un "estado afectivo que preocupa, ya sea por su intensidad, la frecuencias o la cantidad de problemas presentados" (Lucchini, 2002, p. 62), y no una situación aislada.

Estas problemáticas, al impactar desfavorablemente el rendimiento escolar del o la estudiante, presentan como característica fundamental, de acuerdo con el Centro Nacional de Diseminación de Información para Niños con Discapacidades (NICHCY, 2004), su no relación primordial con factores intelectuales, sensoriales o de la salud, sino con una dificultad para formar y mantener relaciones interpersonales con pares y docentes; la cual ésta asociada a comportamientos y sentimientos discordantes en relación con las circunstancias, presentándose un estado general de descontento y tristeza, que puede incluso desarrollar síntomas físicos o temores asociados a problemas personales.

Así, por ejemplo, las dificultades escolares o de aprendizaje, como los problemas de conducta, pueden relacionarse con complicaciones afectivas del niño o la niña, ocasionadas por problemas familiares y sociales, que a la vez pueden generarle otras dificultades para vincularse con otras niñas y niños en la escuela, a manera de patrón relacional, y todo ello se encontrará relacionado, según Trianes y García (2002), con déficits en la madurez y el equilibrio socio- afectivo.

Todo ello ya ha sido contemplado por las bases teóricas de diversas ciencias sociales y de la educación, con autores como Piaget (1995) y Vigotsky (1979), quienes décadas atrás han hecho énfasis en la importancia del entorno familiar y social más inmediato en el desarrollo social y afectivo del niño y la niña, así como la centralidad de las y los otros como importantes referentes para la conformación de la autoestima y el autoconcepto; lo que lleva a la necesidad de que toda intervención a nivel educativo deba tomar en cuenta los factores socio-afectivos que determinan al o la estudiante.

De tal forma, podría considerarse que una o un estudiante tiene un problema socio- afectivo cuando presenta alguna de sus tres principales manifestaciones, las cuales, según Murillo (2005), son:

- Afectación de las relaciones sociales con quienes les rodean y consigo mismo (que pueden llegar a desembocar en impulsividad, agresividad, inatención, uso de vocabulario agresivo). 
- Problemas escolares por estados de ánimo alterados (tristeza, aprehensión, tendencia a desarrollar síntomas físicos o de temor).

- Dificultades para aprender o aprovechar las situaciones de aprendizaje- enseñanza, algunos de ellos no explicables por factores intelectuales, sensoriales o de estado de salud (periodos cortos de atención y concentración, preocupación extrema, carencia de estrategias para estudio y aprovechamiento del tiempo para el mismo, interferencia en el desempeño académico).

Estas son tres de las manifestaciones más comunes mencionadas por el autor, sin embargo la variación de ellas dependerá de los recursos personales y apoyos que cada niña o niño tenga, considerándose, de acuerdo con las y los autores citados hasta ahora, que su determinante fundamental será una problemática social que afecta negativamente el estado emocional del niño o la niña, entre las que resaltan conflictos familiares citados por Espinoza, Herrera y Portuguez (2009), como peleas, separaciones, ausencias y rechazos entre sus miembros.

No obstante, ante una serie de manifestaciones como las antes descritas, siempre ha de ser necesario descartar alguna necesidad especial de mayor compromiso por la existencia de implicaciones de otro tipo que determinan la situación escolar del niño o la niña, donde el factor socio-afectivo no es el principal determinante.

Lo anterior se debe principalmente a que las dificultades escolares relacionadas con problemas socio-afectivos, se consideran esperables cuando son originados "por la vida en extrema pobreza, en carencias afectivo-familiares y/o en la falta de incentivos para aprender que no necesariamente se traducen en patologías pero que predisponen al bajo rendimiento, al fracaso y a la deserción escolar" (Jadue, 2002) y no se consideran tales cuando surgen de factores fisiológicos, genéticos y/o cognitivos.

Concluyendo, que los problemas socio-afectivos, aún cuando puedan carecer de un diagnóstico clínico, "requieren de una atención integral, específica y especializada de profesionales de diversos campos” (Murillo, 2005; p.19).

\section{METODOLOGÍA:}

Para cumplir con el objetivo de esta investigación, se utilizó una metodología mixta con predominancia cualitativa, la cual posibilitó indagar los aspectos socio-afectivos vinculados a las dificultades de éstas y estos estudiantes, de forma que se aplicaron evaluaciones psicológicas que permitieran alcanzar dicho fin. Además de ello, se planteó ésta investigación, como no experimental, descriptiva y transversal.

Sujetos: Las y los estudiantes participantes fueron aquellas/os seleccionados para formar parte del programa, mediante la aplicación de un diagnóstico preliminar por medio de la Prueba Corta, la cual reveló dificultades escolares. Posteriormente se les realizó un 
diagnóstico más completo a través del sistema PEQUES que contempló seis pruebas básicas para determinar problemas en el desarrollo psicomotriz, cognitivo y de las áreas de aprendizaje, quienes dieron resultados negativos, en al menos tres de las pruebas, fueron considerados con riesgo a perder el año escolar, y por tanto seleccionados para participar de la intervención de PSICOMI, a éstas y estos se les conoce como niñas/os “estrella” ${ }^{1}$ y constituyeron así la población de este estudió, siendo tres niñas y tres niños, que en el momento del mismo tenían entre los siete y ocho años, provenientes de primero y segundo grado de la Escuela Villalobos de Lagunilla de Heredia.

Instrumentos: Fueron utilizados el Test de la Figura Humana, de acuerdo al Baremo de Goodenough y la Escala de Weschler (Xándro, 1999), para medir la madurez intelectual del niño o niña, con el fin de conocer sus capacidades y nivel de desarrollo en correspondencia con lo esperado para su edad, ello dentro del enfoque cuantitativo. También fue utilizado en su interpretación proyectiva de acuerdo a los indicadores socio-afectivos de Koopitz (1975), con la cual se analizó el estado emocional del niño y las cargas afectivas dirigidas hacía sí mismo, relacionadas con factores sociales.

Además, se realizó una entrevista abierta la o el encargado del o la estudiante, elaborada en el programa como parte del protocolo del mismo, la cual abarcaba seis áreas principales, que son: cuadro de estructuración familiar, motivo de consulta, situación actual del niño o niña, historia de desarrollo personal del o la misma, conclusiones, recomendaciones e intervención, para finalizar con un apartado de indicaciones para la intervención en PSICOMI.

También fue aplicado el Test del Dibujo de la Familia (Corman, 1967), para analizar la expresión de tendencias inconscientes de las niñas y niños, en cuanto a sentimientos hacia su familia y el lugar que ocupa cada miembro en ella, y fundamentalmente, para conocer el estado emocional del niño o niña con respecto a su medio familiar.

Procedimiento: Tras la selección planteada y los correspondientes permisos de los padres y madres de familia, se midió la madurez intelectual del niño o niña, con el fin de conocer sus capacidades y nivel de desarrollo en correspondencia con lo esperado para su edad, ello dentro del enfoque cuantitativo.

Posterior a ello, se realizaron desde un enfoque cualitativo, las evaluaciones proyectivas, que permitieron identificar cuáles eran las principales problemáticas socio-afectivas de las y los estudiantes que participaron del estudio, así como la entrevista al padre ó madre de familia y la aplicación del Test del Dibujo de la Familia.

Finalmente, para generar mayor confiabilidad se realizó triangulación de resultados a través de instrumentos (tres evaluaciones proyectivas), a la vez que se cuantificaron las tendencias

\footnotetext{
${ }^{1}$ Término que se utiliza para identificar a las niñas y niños que requieren de la intervención en PSICOMI.
} 
comunes de los resultados cualitativos entre las y los estudiantes. Tras la triangulación de las tres pruebas, se logró codificar el contenido para presentar las principales tendencias comunes, logrando una presentación cuantitativa de tales resultados, para posteriormente profundizar en ellos mediante el análisis cualitativo y poder comprender la dinámica de tales factores y su relación con la situación educativa de las y los estudiantes.

\section{Resultados:}

A continuación se presentan los resultados del presente estudio subdivididos en dos categorías de análisis: Madurez infantil y Factores socio-afectivos vinculados con las dificultades escolares, en su presentación cualitativa como cuantitativa.

1. Madurez Infantil de niñas y niños estrella.

La madurez fue evaluada mediante la aplicación del Test de la Figura Humana, calificados a partir del Baremo de Goodenough y la Escala de Wescler.

De esta forma, en la siguiente tabla se muestran los resultados obtenidos de la aplicación de dicha prueba, presentando las edades cronológicas y maduracionales de estas niñas (os), para evaluar si cumplían ó no con las capacidades esperadas para su edad correspondiente.

Tabla1. Madurez infantil según el Test de la Figura Humana

\begin{tabular}{lccl}
\hline Nombre & $\begin{array}{c}\text { Edad } \\
\text { Crono. }\end{array}$ & $\begin{array}{c}\text { Edad } \\
\text { Madur. }\end{array}$ & Madurez \\
\hline A (F) & 7.7 & 9.3 & Supera las capacidades esperadas para su edad \\
S (F) & 8.3 & 7.9 & Presenta el nivel de madurez esperado para su edad \\
D (F) & 8 & 7.9 & Presenta el nivel de madurez esperado para su edad \\
J (M) & 7.3 & 8.9 & Supera las capacidades esperadas para su edad \\
JA (M) & 8.2 & 7.9 & Presenta el nivel de madurez esperado para su edad \\
F (M) & 7.11 & 5.9 & Aún no ha desarrollado el nivel de madurez esperada para su edad. \\
\hline
\end{tabular}

2. Factores Socio-afectivos relacionados a las Dificultades Escolares.

En este apartado se presentan las tendencias comunes, de forma cuantitativa, gracias a una codificación de los datos, así como cualitativa. Para efectos de facilitar la comprensión, se presentarán en primera instancia las tendencias comunes cuantificadas, y posterior a ello a

${ }^{2}$ Las letras $\mathrm{F}$ y $\mathrm{M}$, se refieren al género de cada estudiante, femenino y masculino, respectivamente. 
se profundizará en la dinámica de los factores socio-afectivos y su relación con las dificultades escolares de las y los estudiantes.

a. Tendencias comunes cuantificadas:

Los factores socio-afectivos resaltados por las niñas y niños mediante las pruebas proyectivas, fueron agrupados según tendencias comunes, lo cual se logró mediante la interpretación proyectiva individual, que tras ser realizada reveló puntos sobresalientes compartidos en las historias de las y los estudiantes, permitiendo codificar las tendencias comunes y cuantificarlas, en la siguiente tabla:

Tabla 2.

\begin{tabular}{|c|c|c|c|c|c|c|c|c|c|c|c|c|}
\hline $\begin{array}{l}\text { No } \\
\text { mbr } \\
\text { e }\end{array}$ & $\begin{array}{l}\text { Hijo } \\
\text { (a) } \\
\text { men } \\
\text { or }\end{array}$ & $\begin{array}{l}\text { Conflic } \\
\text { to con } \\
\text { padre }\end{array}$ & $\begin{array}{l}\text { Conflic } \\
\text { to con } \\
\text { madre }\end{array}$ & $\begin{array}{l}\text { Hermana } \\
\text { (o) figura } \\
\text { de } \\
\text { autoridad }\end{array}$ & $\begin{array}{c}\text { Probl. } \\
\text { familiar } \\
\text { es } \\
\text { Graves }\end{array}$ & $\begin{array}{c}\text { Difi. en } \\
\text { el } \\
\text { contacto } \\
\text { social }\end{array}$ & $\begin{array}{c}\text { Demand } \\
\text { a } \\
\text { afectiva }\end{array}$ & $\begin{array}{l}\text { Insegu- } \\
\text { ridad }\end{array}$ & $\begin{array}{c}\text { Agre } \\
\text { si- } \\
\text { vidad }\end{array}$ & $\begin{array}{c}\text { Probl. } \\
\text { académic } \\
\text { os }\end{array}$ & $\begin{array}{c}\text { Proble. } \\
\text { Físicos } \\
\text { y del } \\
\text { desarrol } \\
\text { lo }\end{array}$ & $\begin{array}{c}\text { Deseos } \\
\text { por } \\
\text { cambiar } \\
\text { actual }\end{array}$ \\
\hline A (F) & $\mathrm{X}$ & $\mathrm{X}$ & $\mathrm{X}$ & $\mathrm{X}$ & & $\mathrm{X}$ & $\mathrm{X}$ & $\mathrm{X}$ & $\mathrm{X}$ & $\mathrm{X}$ & $\mathrm{X}$ & $\mathrm{X}$ \\
\hline $\mathrm{S}(\mathrm{F})$ & $\mathrm{X}$ & $\mathrm{X}$ & $\mathrm{X}$ & $\mathrm{X}$ & $\mathrm{X}$ & & $\mathrm{X}$ & $\mathrm{X}$ & $\mathrm{X}$ & $\mathrm{X}$ & & $\mathrm{X}$ \\
\hline $\mathrm{D}(\mathrm{F})$ & $\mathrm{X}$ & $\mathrm{X}$ & $X$ & $X$ & $X$ & $X$ & $X$ & $X$ & $X$ & $X$ & $X$ & $X$ \\
\hline $\mathrm{J}(\mathrm{M})$ & $X$ & $\mathrm{X}$ & $\mathrm{X}$ & & $\mathrm{X}$ & $\mathrm{X}$ & $X$ & $X$ & $\mathrm{X}$ & $X$ & $\mathrm{X}$ & $X$ \\
\hline $\begin{array}{l}\mathrm{JA} \\
\text { (M) }\end{array}$ & $\mathrm{X}$ & $\mathrm{X}$ & & $\mathrm{X}$ & & $\mathrm{X}$ & $\mathrm{X}$ & $\mathrm{X}$ & & & $\mathrm{X}$ & \\
\hline $\begin{array}{c}\mathrm{F} \\
(\mathrm{M})\end{array}$ & $X$ & $\mathrm{X}$ & X & & X & X & X & & & $\mathrm{X}$ & X & \\
\hline $\begin{array}{c}\text { Tot } \\
\text { al }\end{array}$ & 6 & 6 & 5 & 4 & 4 & 5 & 6 & 5 & 4 & 5 & 5 & 4 \\
\hline$\%$ & $\begin{array}{c}100 \\
\% \\
\end{array}$ & $100 \%$ & $84 \%$ & $68 \%$ & $68 \%$ & $84 \%$ & $100 \%$ & $84 \%$ & $68 \%$ & $84 \%$ & $84 \%$ & $68 \%$ \\
\hline
\end{tabular}

Factores socio- afectivos agrupados por tendencias comunes.

(F): Femenino (M): Masculino (X): Presencia de dicho factor

Revista MHSalud® (ISSN: 1659-097X) Vol. 9. No. 1. Enero-Julio, 2012

https://doi.org/10.15359/mhs.9-1.2 
De acuerdo con la tabla 2, cada tendencia fue compartida por más del $50 \%$ de las y los niños, es decir al menos tres de los seis casos presentaron factores socio-afectivos comunes, lo que da luz de un perfil psicosocial de las niñas y niños “estrella”.

La siguiente lista detalla un poco más los factores socio-afectivos comunes, encontrados en las niñas y niños que conformaron el estudio:

- Hija/o menor.

- Demanda afectiva.

- Relación conflictiva con el padre (violencia, distanciamiento o permisividad).

- Relación conflictiva con la madre (dependencia, violencia u hostilidad).

- Dificultades en el contacto social (dependencia, agresividad y/o problemas de comunicación).

- Inseguridad (con respecto a sí mismo, a las habilidades para el estudio o con respecto a la familia).

- Dificultades escolares (inseguridad, no les gusta y/o problemas de lenguaje).

- Problemas físicos o de desarrollo (motrices, sobrepeso y/o lenguaje).

- Hermana/o mayor como figura de autoridad, la o el que le provoca ansiedad o protección.

- Problemas Familiares Graves (Alcoholismo, violencia y probl. Conyugales)

- Agresividad (en la familia, hacia sí mismo y en el contacto social)

- Deseos por Cambiar el Estado Actual de las Cosas

El siguiente gráfico muestra la incidencia de dichos factores, donde el 100\% representa a la totalidad de niñas/os, el $84 \%$ a cinco de ellas y ellos, y el 68\% a cuatro niñas/os.

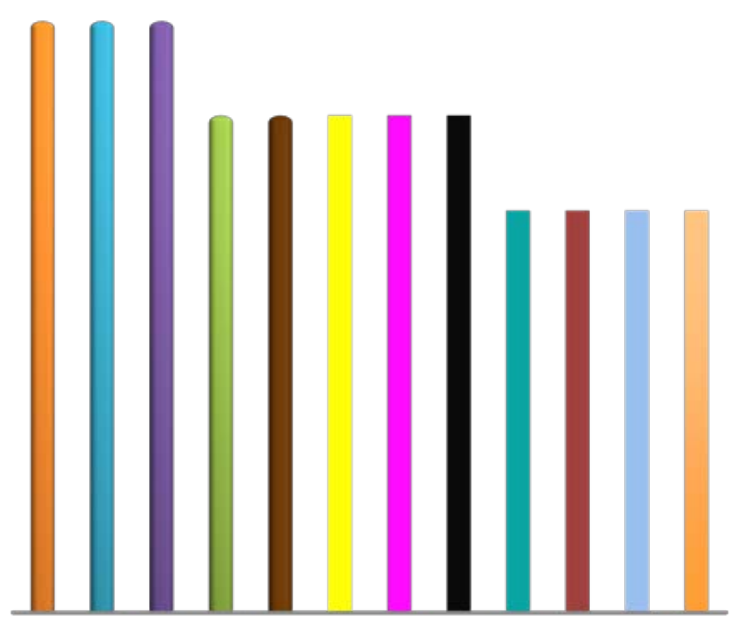

$\square$ Hijo (a) menor

\. Demanda Afectiva

■elación Conflictiva con el Padre

$₫$ Relación Conflictiva con la Madre

口 Dificultades en el Contacto Social

Inseguridad

n Dificultades escolares

- Problemas Físicos o de Desarrollo

- Hermano (a) mayor como Figura de Autoridad

Gráfico1. Incidencia de Factores socio-afectivos comunes. 
b. Factores socio-afectivos y dificultades escolares: resultados cualitativos.

Las tablas anteriores grafican los resultados obtenidos mediante la interpretación proyectiva, sin embargo, a través de la misma es posible profundizar aún más en la dinámica de tales factores en la vida de cada niña ó niño, alcanzando resultados reveladores, los cuales fueron agrupados en tres categorías generales para presentarse a continuación:

\section{Factores a nivel familiar}

Las niñas y niños resaltaron problemáticas sociales importantes relacionadas con el entorno familiar donde se desenvuelven, tales como el alcoholismo, la violencia intrafamiliar y de pareja, madres y padres distantes, además de la separación entre ambos, que afecta emocionalmente a las y los estudiantes.

Asimismo, en las evaluaciones, las y los estudiantes representaron el silencio que mantienen ante las situaciones familiares que les son emocionalmente fuertes, en tanto se manifiestan imposibilitados para poder manejarlas y solucionarlas dentro de su entorno familiar y no poder hablarlas fuera de él. De igual manera, reflejaron un manifiesto deseo por cambiar el estado actual de las cosas.

Es sobresaliente, como factor común, que en las dinámicas familiares de las y los niños, se presentan tres variaciones en los roles familiares tradicionales. En primer lugar, la función del padre es en la mayoría de las ocasiones distante, sino ausente, violento y en pocos casos, contrariamente excesivamente permiso y endeble, lo que resulta afectar emocionalmente a las y los niños.

En un segundo rol variante, se presenta en el 68\% de los casos, la existencia de un hermano mayor con una función de autoridad, ya fuese hostil o por el contrario protectora, pero que en sí constituía un referente sumamente significativo para ellas y ellos.

Por su parte, en tercer lugar, un rol significativo para las y los niños, fue el referido a la madre, con respecto a la cual, las y los estudiantes presentaron en cinco de los seis casos, dependencia materna, asociada a una marcada demanda de afecto que ellas y ellos recalcan, además de la inestabilidad e inseguridad que sienten sobre sí mismos.

Esto se acompaña de una sobresaliente tendencia común, referida a que en su totalidad, las y los niños son hijos menores.

\section{Factores a nivel escolar y en la interacción con pares}

Las vinculaciones con pares ó en la escuela, se mostraban en su gran mayoría conflictivas, mientras que para las y los niños, en su mayoría, la escuela significó un mecanismo de escape y huida de las problemáticas familiares.

Consecuentemente, en cuatro de los seis estudiantes, se presentó inhibición y distanciamiento de las y los demás, de los dos estudiantes con tendencia a la 
extroversión, uno de ellos es agresivo en el contacto con las y los otros, y la última estudiante es dependiente emocionalmente de su familia y amigas.

Por su parte, en cuanto a problemas académicos percibidos por las y los estudiantes, sobresale la inseguridad en las capacidades propias para desarrollarse académicamente, así también, desarrollados por el disgusto hacia algunas materias escolares, pero según relataron las madres en la entrevista, esta situación ha mejorado gracias a la intervención en PSICOMI.

De igual forma, se presentan problemas escolares debidos a problemas de lenguaje presentados en dos estudiantes. Es importante rescatar que todos los problemas emocionales presentados, en esta como en las demás categorías, afectan el área educativa de la vida de las y los estudiantes.

\section{Factores a nivel personal}

Con respecto al nivel de autoevaluación, la inseguridad en sí mismos, en sus capacidades físicas, intelectuales y generada por problemas familiares, es una tendencia común, así como también lo es la agresividad, que se presento en dos direcciones, en uno de los casos hacia el entorno social, mientras en las más de las veces estuvo dirigida hacia el sí mismo, como agresividad autodestructiva hacia rasgos propios que consideran negativos, en ambos casos vinculada a los problemas familiares, por ejemplo en el caso de una estudiante que comía ansiosamente porque se sentía culpable por la ausencia de su padre.

Por su parte, es un factor común a las y los estudiantes la tendencia a la inhibición, la cual junto a la agresividad en el contacto y dependencia en el mismo, en el caso de un niño y una niña extrovertidos, se relacionaron con la manifestación de sensaciones de inestabilidad y desequilibrio en sus situaciones de vida.

Entre ellas y ellos, en su totalidad, se muestra una dificultada para nivelar el control de los impulsos versus la racionalidad, sin embargo, este rasgo se considera esperable para la etapa del desarrollo en que se encuentran, pues esta capacidad se desarrolla con mayor facilidad posterior a la adolescencia.

De igual forma, asociado a su edad y etapa de desarrollo, se presenta como rasgo común la vitalidad y potencia física, siendo esta la capacidad por medio de la cual se trabaja sesión a sesión dentro del Programa Psicomotricidad e Intervención. La misma es importante pues, si se potencia una habilidad que ellas y ellos mismos perciben, se podrá mejorar el autoconcepto, el autoestima, la seguridad en sí mismos, el sentido de independencia y consecuentemente el establecimiento de relaciones sociales.

\section{Discusión:}

Con respecto al nivel de madurez, es importante mencionar que en el momento en que se realizaron las evaluaciones, las niñas y niños ya habían pasado por un proceso de intervención en PSICOMI, lo que pudo influir en la mejora de habilidades motrices, 
cognitivas e incluso en sociales, al integrar a las madres y padres de familia en la intervención, con el objetivo de potenciar el desarrollo integral de sus participantes.

Por otra parte, entendiendo que la madurez se refiere en este caso, como sugiere Watson (2005), a la posibilidad de que una o un estudiante, en un momento dado de su etapa escolar, posea un nivel de desarrollo físico, psicológico y social que le permite enfrentar adecuadamente a las situaciones que esa etapa le presenta, así como a las exigencias asociadas a ella, junto con la posibilidad de apropiarse de los valores tradicionales y de la cultura de su entorno, se pude afirmar que:

- En general, las niñas y niños evaluados presentan un nivel de madurez físico, psicológico y social que les permita enfrentarse a su situación escolar, es decir, tienen las capacidades necesarias para hacerlo, sin embargo, de acuerdo a las evaluaciones realizadas en PSICOMI, siguen presentando dificultades a nivel escolar.

- Hay un estudiante que no cumple de forma esperable con el nivel de madurez para su edad, no obstante, tal nivel puede verse influenciado por las dificultades en la salud, que ha presentando el menor desde que padeció meningitis en su primer año de edad, con secuelas tales como problemas de lenguaje.

Lo anterior comprueba que, como afirma Watson (2005), no existe un estado de madurez general que garantice el éxito en el dominio de la situación escolar, si no que existen niveles de desarrollo de funciones susceptibles de ser mejoradas si se respeta el tiempo en que deben ser enseñadas, así como las formas de efectuar las actividades escolares.

Con ello se constata, como se mencionó con anterioridad, que existen dificultades escolares que no se encuentran asociadas a la madurez del estudiante, sino que muchas veces pueden encontrarse afectadas por factores socio-afectivos negativos que les impiden a las niñas y niños poder desarrollarse de la forma esperada.

Siendo así, es fundamental identificar cuales factores socio-afectivos podrían verse involucrados, ya que desde la perspectiva de la intervención psicomotriz, el desarrollo es integral, y por tanto, en menor o mayor medida, los problemas de ésta índole podrán ir limitando las posibilidades posteriores de desarrollo de estas niñas y niños, y ha de ser necesario intervenir en ese punto.

Al realizar tal análisis, se comprobó que de manera dialéctica, el entorno social y ambiental en que se desenvuelve el niño o la niña, determina sus oportunidades de desarrollo, brindándole las herramientas necesarias o no para que alcance el máximo de sus capacidades.

En cuanto a su entorno familiar, se evidencia que los factores relacionados con ésta, constituyen el área más influyente en el estado emocional de las y los niños, ya que se reveló, una mayor presencia de situaciones familiares conflictivas y desgastantes, las cuales, ellas como ellos, vincularon con las demás áreas de su vida, por ejemplo la escolar. 
Asimismo, el silenciar las emociones y las situaciones que viven, se convierte en un mecanismo de represión, sin embargo, el impacto de tales experiencias es grande e intolerable, tendiendo, posiblemente, a manifestarse a través de otras problemáticas como lo son las dificultades escolares.

Evidentemente, un ambiente familiar cargado de conflictos, como separación de padres, violencia, hostilidad e indiferencia, provoca déficit en el desarrollo emocional, lo cual es reforzado por Jadue (2003) al afirma que existen diversos estudios que han mostrado que los niños con buenas relaciones con sus padres van mejor a nivel educativo, mientras que las experiencias familiares negativas afectan el autoconcepto, su capacidad de control emocional y conductual, y sus procesos cognitivos, además de generarles mucha ansiedad.

Además, fue evidentemente particular la función del rol paterno, que en el caso de estas y estos estudiantes, se torno agresivo, distante o permisivo, lo que estructuró una relación conflictiva con la niña o el niño, propiciándole una base poco firme que le brinde seguridad para desarrollarse y confiar en sí mismas o mismos. A la vez, tal situación provoca la demanda afectiva y el establecimiento de la dependencia materna, donde el apego funge como mecanismo para asegurarse y sostenerse de una persona adulta, aunque, también se presentaron conflictos con la madre. Esta situación lleva a la ubicación de un hermano (a) mayor como figura de autoridad.

Para el niño o niña, también es conflictivo que su hermano (a), que se supone en una relación de horizontalidad, sea quien figure como autoridad, lo que le genera cierta inestabilidad, aún cuando la alternativa de protección que éste o ésta le brinda suele darle cierto grado de compensación ante la falta de la función paterna y materna.

Sin embargo, tal situación no soluciona su inestabilidad emocional ni suministra la seguridad posible y necesaria de parte de la persona adulta, ya que como hermana/o no está en disponibilidad para cumplir tal función. Además, porque existieron casos en que tal hermano (a) incremento la hostilidad, tornando la situación afectiva aún más tensional y difícil para el niño o la niña.

De manera tal, como afirma la autora, "las dimensiones positivas o negativas de su relación con cada padre, son predictores de la adaptación a la escuela” (Jadue, 2003), por lo que las dificultades educativas que enfrentan las y los niños "estrella” son esperables ante las situaciones tan difíciles que enfrentan para una edad tan temprana.

Así es que, el estar inmersos en situaciones de violencia y alcoholismo dentro del entorno familiar, crea tensiones y presiones para las que un niño o niña, sin importar su nivel maduracional, no está preparado (a) para enfrentar, por lo que se ven afectadas todas las áreas de su vida, intermediadas por su estado emocional.

Si a dichas problemáticas se suma el hecho de ser el hijo o hija menor, que se caracteriza por ser una posición y lugar en la familia donde existe mucha labilidad y dependencia, se vuelve esperable que las presiones a las que están sujetas y sujetos 
sobrepasen sus recursos personales, por lo que tal imposibilidad sentida para solucionarlas, les lleva a tener consecuencias manifiestas en su ámbito escolar.

Así, entre los factores socio-afectivos más influyentes se encuentran los conflictos familiares que les generan sentimientos negativos con respecto a sí mismos, en tanto el desempeño escolar y la interacción con las y los pares se ven consecuentemente afectadas por ello.

De forma que, las problemáticas que empiezan a sobrevenir en el ámbito escolar, ya sea por dificultades académicas o enfrentamientos con pares, vuelven al que eventualmente sería un ámbito seguro y alternativo, en un lugar a donde se desplazan las tensiones. Es por ello que, dentro de la intervención psicomotriz ha de procurarse crear un ambiente seguro, en el cual el niño o niña pueda encontrar una real alternativa, no para huir, sino para enfrentar, con el sostenimiento adulto necesario, las problemáticas que le aquejan y atormentan.

Todo esto revela la evidente afectación del niño o niña como tal y su percepción y estima hacia sí mismo. Con respecto a ello, la tendencia a la inhibición, se torna un mecanismo defensivo, a manera de represión, ante situaciones en su entorno que consideran amenazantes, como las ya mencionadas, que les vienen ubicando en un lugar de inestabilidad.

La tendencia en la dificultad para la verbalización de las emociones parece deberse tanto a la tensión que pueden causarles el cargar dichas emociones, así como la dificultad que requiere para una niña o un niño entenderlas y darles sentido. No obstante, tras ello, permanece latente un deseo manifiesto por cambiar el estado actual de sus situaciones de vida, mostrando su inconformidad, ante la cual no consideran tener el poder suficiente para modificarlas solos.

No obstante, a pesar de considerarse, como niños o niñas, incapaces para resolver por sí solos las problemáticas en las cuales se encuentran inmersos, lo cual parece ser una consideración acertada, existe una adecuada valoración de sus capacidades para su edad, entre las cuales resaltan su vitalidad y potencia física, mediante la cual se trabaja sesión a sesión dentro del Programa Psicomotricidad e Intervención. La misma es importante pues, si se potencia una habilidad que ellas y ellos mismos perciben, se podrá mejorar el autoconcepto, el autoestima, la seguridad en sí mismos, el sentido de independencia y consecuentemente el establecimiento de relaciones sociales, indirectamente mejorando el enfrentamiento de tales problemáticas.

Con todo ello, sí como dice Jadue (2003) los estudiantes con buen autoconcepto, expectativas positivas de su rendimiento y una motivación intrínseca para aprender, obtienen mejores logros en la escuela, es de esperarse que niñas y niños que por el contrario, son inseguros de sus capacidades escolares, se siente inhibidos y frágiles y no sienten agrado por la escuela (dadas sus conflictos familiares), tengan dificultades en la misma, como se comprobó con las y los niños “estrella”. 
De tal forma, tratando de entrelazar todo lo hasta ahora expuesto, al relacionar los resultados obtenidos sobre la madurez, los factores socio-afectivos y las dificultades escolares por las cuales las y los niños fueron llevados a PSICOMI, se constata que:

Las situaciones familiares difíciles pueden ser un obstáculo que los niños al deber enfrentar, lo cual puede obligarles a desarrollar capacidades cognitivas y maniobras emocionales, para sobreponerse a ello y seguir su proceso de desarrollo, por lo que, aún siendo situaciones difíciles, han estimulado que estos niños y niñas desarrollen un nivel de madurez mayor para lograr enfrentarlas.

A pesar de ello, enfrentar situaciones socio-afectivas conflictivas y difíciles para ser resueltas o atendidas a su edad, puede ser el factor determinante para que ellas y ellos, a largo plazo puedan, por el contrario, ver limitado el alcance de un mayor nivel de madurez, convirtiéndose un obstáculo para un desarrollo y crecimiento óptimo.

Es por ello que, aun considerando que las y los estudiantes tienen las capacidades necesarias para enfrentar las situaciones comunes para su edad, como el ingreso y adaptación a la escuela, el establecimiento de relaciones sociales con los pares, entre otras, parece ser que las situaciones socio- afectivas a las que se enfrentan, están determinado su desempeño educativo de forma negativa, pues aún teniendo un nivel idóneo de desarrollo, siguen presentado dificultades académicas.

\section{CONCLUSIONES:}

Las niñas y niños participantes del estudio, en general, poseen un nivel de madurez óptimo para enfrentar los retos esperadas para su edad, sin embargo, las presiones y situaciones, principalmente de orden familiar, sobrepasan sus recursos personales y exigen de ellos capacidades que aún no les es posible desarrollar, por lo que ante tal tensión, se ve afectado su estado emocional, repercutiendo en todos los ámbitos en que el niño o niña se desenvuelve, tal como el escolar.

Así, se comprueba que "las emociones mediatizan la capacidad de adaptarse y de responder a una variedad de experiencias" (Jadue, 2002), involucrándose profundamente en la dirección de procesos cognitivos como la atención, percepción, aprendizaje y memoria, necesarios en el procesos de aprender, sin necesariamente encontrarse algún compromiso físico o cognitivo.

Finalmente, lo que este estudio comprueba es que el niño y la niña, en su desarrollo integral, se encuentra indiscutiblemente influido por el entorno en que se desenvuelve, $y$ por tanto, en este caso, la situación familiar conflictiva fue determinante en el estado emocional del niño o la niña, con respecto a sí mismo y su entorno, generándole dificultades escolares y sociales. Por ello, la necesidad de intervenir en estas dificultades a un nivel que trascienda la dificultad escolar, que es solo la manifestación de un conflicto, y contemple la amplitud del individuo, llegando hasta el nivel socio- afectivo, lo que lleva, "hoy en día se reconoce que tanto las familias como los niños necesita apoyo" (NICHCY, 2004; p.4) pues la sola intervención con él o la estudiante no ha de ser suficiente para mejorar su situación socio- afectiva, ya que no tienen el grado de 
independencia necesario para alejarse de un entorno que le afecta negativamente, y en ello PSICOMI ha tomado parte.

\section{REFERENCIAS:}

Centro Nacional de Diseminación de Información para Niños con Discapacidad. (2004). Hoja Informativa sobre Discapacidades, Problemas Emocionales. Washington, Estados Unidos. Recuperado desde: www.nichcy.org

Corman, L. (1967). El Test del Dibujo de la Familia. Buenos Aires, Argentina: Kapelusz.

Espinoza, G., Herrera, E., \& Portugués, V. (2009). Análisis de los factores socioafectivos y pedagógicos que influyen en los procesos de aprendizaje en los niños y niñas identificados (as) como estrellas por el programa PEQUES del Centro Educativo Villalobos, en Lagunilla de Ulloa de la provincia de Heredia, Circuito Escolar 02 (Tesis para optar por el grado de Maestría en Psicopedagoía, Universidad Estatal a Distancia). San José, Costa Rica: UNED.

Estado de la Nación. (2009). Estadísticas Sociales. Educación. Recuperado desde: http://www.estadonacion.or.cr/index.php/estadisticas/costa-rica/compendioestadistico/estadisticas-sociales

Jadue, G. (2002). Factores Psicológicos que predisponen al Bajo Rendimiento, al Fracaso y a la Deserción Escolar. Estudios Pedagógicos, (8), 193-204.

Recuperado desde:

http://www.scielo.cl/scielo.php?script=sci_arttext\&pid=S0718-

07052002000100012\&lng=en\&nrm=iso\&ignore $=. \mathrm{html}$

Jadue, G. (2003). Transformaciones Familiares en Chile: Riesgo Creciente para el Desarrollo Emocional, Psicosocial y la Educación de los Hijos. Rev. Estudios Pedagógicos, (29), 115-126. Recuperado desde: http://www.scielo.cl/scielo.php?pid=S071807052003000100008\&script=sci_artt ext

Koopitz, E. (1975). El Dibujo de la Figura Humana en los Niños. Buenos Aires: Editorial Guadalupe.

Lucchini, G. (2002). Niños con Necesidades Educativas Especiales. Ediciones Universidad Católica de Chile.

Murillo, A. (2005). Normas y Procedimientos para el Manejo Técnico- Administrativo de los Servicios Educativos para Estudiantes con Problemas Emocionales y de Conducta. Centro Nacional de Recursos para la Inclusión Educativa. Ministerio de Educación Pública. San José, Costa Rica.

Piaget, J. (1995). Seis estudios de Psicología (5ed). Colombia: Grupo Editor Quinto Centenario Colombia. 
URL www.una.ac.cr/mhsalud

Vygotsky, L. (1979). El Desarrollo de los Procesos Psicológicos Superiores. Buenos Aires, Argentina: Grijalbo.

Watson, R. (2005). Diagnóstico Educacional y Vocacional. Universidad Católica del Norte.

Woodburn, S., Fernández, H., \& Boschini, C. (2007). El Diagnóstico Educativo del Fracaso Escolar: el Sistema Peques. Heredia, Costa Rica.

Fecha de recepción: 16 de mayo de 2012.

Fecha de aceptación: 11 de junio de 2012.

Fecha de publicación: 31 de julio del 2012. 